\title{
Effects of Pregabalin on Postnatal Development of Kidney in Albino Rat's Offsprings
}

\author{
Hala H. M. Mossalam* MD
}

\author{
* Corresponding Author: \\ Hala Mossalam \\ halahamed1000@gmail.com
}

Received for publication July 5 , 2021; accepted August 12, 2021; published online August 12, 2021.

Copyright 2021 The Author published by Al-Azhar University Faculty of Medicine, Cairo, Egypt. All rights reserved. This an openaccess article distributed under the legal terms, where it is permissible to download and share the work provided it is properly cited. The work cannot be changed in any way or used commercially.

\section{doi:10.21608/aimj.2021.80323.1502}

*Department of Anatomy and Embryology, Faculty of Medicine for Girls. Al-Azhar University.

\begin{abstract}
Background: Pregabalin is used for treatment of neuropathic pain, fibromyalgia, and migraine.

Objectives: This study was designed to assess the effect of pregabalin on the developing kidney of albino rat's offspring.

Material and methods: This study was conducted between September 2019 and January 2020, at Faculty of Medicine for Girls, Al-Azhar University. Twenty pregnant rats were divided into two equal groups: group I (control) group and group II (treated) group which received pregabalin $(150 \mathrm{mg} / \mathrm{kg} / \mathrm{day})$ from the first day of gestation up to 21 days after delivery. Offsprings were selected to be sacrificed at the $2^{\text {nd }}$ postnatal day and at the $21^{\text {st }}$ postnatal day, kidney specimens were prepared for light and electron microscopic examination.

Results: Maternal pregabalin administration disrupted the development of the kidney in offsprings with a significant increase in urea and creatinine level. Histological examination of the kidney offsprings at the $2^{\text {nd }}$ postnatal day revealed irregular thickening of the glomerular basement membrane, fusion, and effacement of podocyte's foot processes and tubulointerstitial injury. Examination of the kidney offsprings at the $21^{\text {st }}$ postnatal day revealed thickened distorted glomerular basement membrane, broadening, and fusion of the foot processes and vacuolar degeneration of tubules.

Conclusion: Maternal pregabalin might disturbed the renal development in the offspring. So, it should be used with caution during pregnancy and lactation.
\end{abstract}

Keywords: Kidney development; Pregabalin; Rat.

\section{INTRODUCTION}

Anti-epileptic drugs (AEDs) have the possibility of affecting the fetal development throughout pregnancy ${ }^{1}$. Many AEDs have been shown to readily cross the placental barrier reaching the fetal circulation causing major and minor teratogenic effects ${ }^{2}$. The new AEDs as pregabalin, felbamate, gabapentin, lamotrigine, levetiracetam, topiramate, etc., have been launched for clinical usage whereas, clinical data on their teratogenic adverse effects are still limited ${ }^{3}$. Pregabalin is the latest compound that joins the list of approved new AEDs that shows efficacy in the treatment of uncontrolled partial seizures, neuropathic pain, generalized anxiety disorder, fibromyalgia, sleep disorders, and migraine ${ }^{4}$. Pregabalin is a gamma-amino butyric acid (GABA) analogue that acts by increasing neuronal GABA level ${ }^{5}$. It is structurally related to gabapentin, but it has the benefit of more efficacy and absorption ${ }^{6}$.

\section{MATERIAL AND METHODS}

Animals: The animals were housed in the animal facility at the Faculty of Medicine for Girls, Al-Azhar University. This study was conducted between September 2019 and January 2020, on twenty pregnant rats in accordance with ethical procedures and policies approved by Animal Care and Use Committee of Faculty of Medicine, Al-Azhar University, Cairo, Egypt.

The pregnant rats were divided into two equal groups: Group I (Control group): composed of 10 pregnant female rats that received distilled water. Group II (treated group): composed of 10 pregnant female rats that were given pregabalin drug (150 $\mathrm{mg} / \mathrm{kg} /$ day) from the first day of gestation and sustained up to 21 days after delivery. Offsprings included in the present study were sacrificed at the $2^{\text {nd }}$ postnatal day and $21^{\text {st }}$ postnatal day. The 
offsprings were divided into four groups (10 animals each):

Group (1): composed of control male offspring at the $2^{\text {nd }}$ postnatal day.

Group (2): composed of treated male offspring at the $2^{\text {nd }}$ postnatal day.

Group (3): composed of control male offspring at the $21^{\text {st }}$ postnatal day.

Group (4): composed of treated male offspring at the $21^{\text {st }}$ postnatal day.

Chemicals: Pregabalin capsules (Lyrica $\left.{ }^{\circledR}\right) 300 \mathrm{mg}$ Pfizer-Egypt Company was purchased. The dose used was the minimal human therapeutic dose (150 $\mathrm{mg} / \mathrm{kg} / \mathrm{day})^{7}$. The equivalent rat dose was calculated by using the formula of Paget and Barens ${ }^{8}$.

Methods: Light microscopic examination, the right kidney was processed then stained with haematoxylin and eosin (H\&E) ${ }^{9}$. Transmission electron microscopic (TEM) examination, the left kidney was processed ${ }^{10}$ and examined by transmission electron microscope (JEOL1010, Japan) at the Regional Centre for Mycology and Biotechnology (RCMB)Al-Azhar University, Cairo.

Blood samples were collected from the retro-orbital sinus of each offspring by a fine capillary glass tube then centrifuged. Sera were collected for assessment of serum urea and creatinine then were carried out ${ }^{11}$ and ${ }^{12}$ at the Biochemistry Department, Faculty of Medicine, Cairo University. Statistical analysis of urea and creatinine in all studied groups was carried out using the SPSS statistical package. The collected data were analysed (Expressed as means \pm SD) and statistical significance was performed using one-way ANOVA followed by a Tuckey post-Hoc test for multiple comparisons. So, Values o $\mathrm{P} \leq 0.05$ were statistically significant ${ }^{13}$.

\section{RESULTS}

Light microscopic examination:

Group (1): revealed that the kidney was formed of an outer cortex and inner medulla with medullary rays passing through the cortex and medulla dividing it into renal lobules. The renal cortex showed two cortical zones, the subcapsular nephrogenic zone and the juxtamedullary zone. In the subcapsular nephrogenic zone, immature forms of renal glomeruli, which include cell clusters, ureteric buds, hemispherical glomeruli, comma-shaped and Sshaped glomerular structures close to the capsule surrounded by primitive convoluted tubules. The juxtamedullary zone contained more mature renal corpuscles, convoluted tubules, and interstitial tissues (Fig.1a). In the juxtamedullary zone, the renal corpuscle was formed of a glomerulus (glomerular capillary tuft) surrounded by Bowman's capsule. The Bowman's capsule was formed of parietal layer lined by flat squamous epithelium and visceral layer closely applied to the glomerular capillaries with Bowman's space in between. Proximal convoluted tubules (PCTs) were lined by cubical cells with oval basal nuclei and acidophilic granular cytoplasm with a narrow lumen. Distal convoluted tubules (DCTs) were lined by simple cuboidal epithelial cells with central rounded nuclei with wide lumina (Fig. 1b).

Group (2): the kidney was divided into the outer cortex and inner medulla with faint medullary rays. The renal cortex showed crowded immature renal glomeruli with primitive tubules (Fig.1c), while the deeper cortex showed glomerular and tubulointerstitial injury. The glomeruli appeared distorted, congested with a widening of Bowman's space. In addition, there was dilatation in the PCTs and DCTs lumina as well as degeneration and vacuolation associated with exfoliation of their epithelial lining. The interstitial tissues revealed haemorrhage with congested peritubular capillaries in between the renal tubules (Fig.1d).

Group (3): the kidney acquired full maturation and differentiated into an outer cortex and the inner medulla. The renal cortex showed numerous uniform renal corpuscles, PCTs, DCTs surrounded by interstitial tissues with disappearance of most of the immature forms (Fig.2a). The renal corpuscles consisted of renal glomeruli surrounded by Bowman's capsules which consisted of parietal and visceral layers. The parietal layer was formed of flat cells which were separated by the Bowman's space from the visceral layer which was closely applied to the renal glomeruli. PCTs were lined by cuboidal cells with oval basal nuclei and acidophilic cytoplasm with a narrow lumen. DCTs were lined by cuboidal cells with large apical vesicular nuclei and acidophilic cytoplasm with wide lumina (Fig.2b).

Group (4): the kidney was differentiated well into outer cortex and inner medulla with the disappearance of most of the immature forms. The renal cortex exhibited disturbed architecture with congested shrunken glomeruli, wide Bowman's space with interstitial congestion and extravasation of blood (Fig.2c). Dilatation in the PCTs and DCTs lumen with degeneration and vacuolation in their epithelial lining with congested peritubular capillaries. (Fig.2d). Transmission electron microscopic examination: Group (1): the renal corpuscles formed of glomerular blood capillaries which may contain red blood cells (RBCs). The glomerular blood capillary was formed by a thin glomerular basement membrane lined by endothelial cells and surrounded by podocytes and mesangial cells. Endothelial cells had euchromatic nuclei with cytoplasmic fenestrations. The podocytes had large euchromatic nucleus, abundant cytoplasm, with primary foot processes that split up into numerous secondary foot processes with filtration slits in-between (Fig. 3a \& 4a).

The PCT cells had oval basal nuclei resting on a basement membrane with dense apical microvilli. The cytoplasm immediately below the microvilli contained many pinocytic vesicles, numerous mitochondria, and few lysosomes (Fig.5a).

DCT cuboidal cells had large oval euchromatic nuclei, numerous mitochondria and short apical microvilli and wide lumen (Fig.6a). 
Group (2): the renal corpuscle had thickened irregular glomerular basement membrane. The endothelial cells had a large, intended nucleus with peripheral chromatin condensation. The podocyte had distorted primary foot processes with fusion and effacement of its secondary foot processes with narrow obliterated filtration slits (Fig. $3 b \quad \& \quad 4 b$ ). The PCT cells had irregular nuclei, cytoplasmic vacuolations, and few mitochondria with many lysosomes (Fig. 5b). The DCT cells had shrunken nuclei, cytoplasmic vacuolations and few mitochondria (Fig. 6b).

Group (3): the glomerular capillary formed by thin glomerular basement membrane which was lined with fenestrated cytoplasm of endothelial cells. The podocyte had a large nucleus with primary and secondary foot processes (Fig. 3c) and (Fig. 4c). The PCT columnar cells resting on a basement membrane with oval euchromatic nuclei with dense apical microvilli that filled most of the lumen. The cytoplasm contained many pinocytic vesicles, numerous mitochondria, and few lysosomes (Fig.5c). The DCT cuboidal cells had rounded nuclei, numerous mitochondria and few microvilli projecting into the well-defined lumen (Fig.6c)

Group (4): showed the glomerular blood capillary containing red blood cell with thickened distorted glomerular basement membrane. The endothelial cells had large, intended nucleus with peripheral chromatin condensation. The podocyte had distorted primary foot processes, with broadening and fusion of secondary foot processes (Fig.3d \&4d). The PCT cells had irregular intended nuclei, pinocytic vacuoles, few mitochondria, and many lysosomes (Fig.5d). The DCTs cells had irregular nuclei, cytoplasmic vacuolations and few mitochondria (Fig.6d).

\section{Biochemical study:}

The data illustrated in table (1),(2) and figures (7) ,(8) revealed a significant increase in the levels of serum urea and creatinine in group (2) and (4) if compared with group (1) and group (3) respectively.

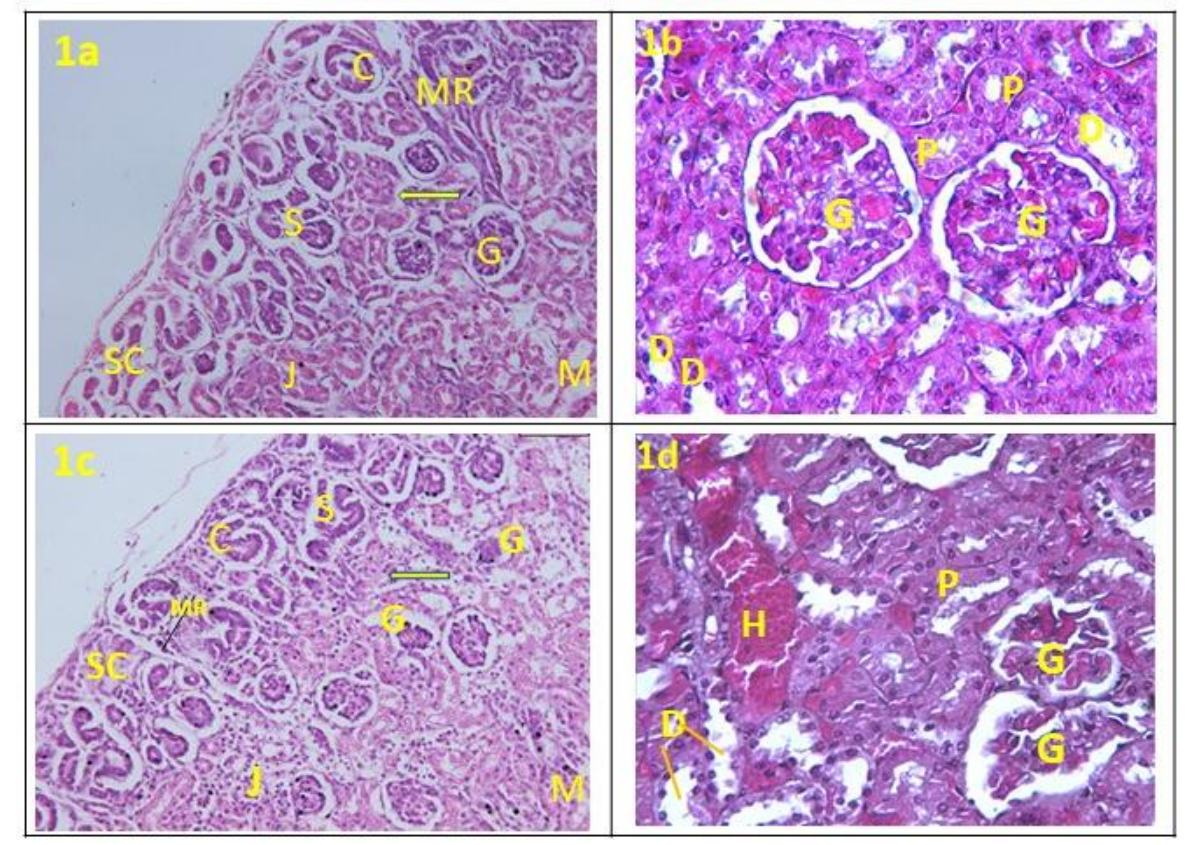

Fig. 1: Light photomicrographs of:

a: Group (1) showing subcapsular nephrogenic zone (SC), juxtamedullary zone (J), medulla (M) medullary rays (MR), hemispherical glomeruli(G), comma-shaped bodies (C), S-shaped bodies (S), primitive tubules (arrow) (H\&E×200).

b: Group (1) showing glomeruli (G), proximal (P), distal (D) convoluted tubules (H\&E×400).

c: Group (2) showing subcapsular nephrogenic zone (SC), juxtamedullary zone (J), medulla (M), faint medullary rays (MR), distorted glomeruli (G), comma-shaped (C), S-shaped bodies (S), primitive convoluted tubules (arrow) $(\mathrm{H} \& \mathrm{E} \times 200)$.

d: Group (2) showing distorted glomeruli $(\mathrm{G})$, haemorrhage $(\mathrm{H})$, exfoliated proximal $(\mathrm{P})$ and distal (D) tubules (H\&E×400). 


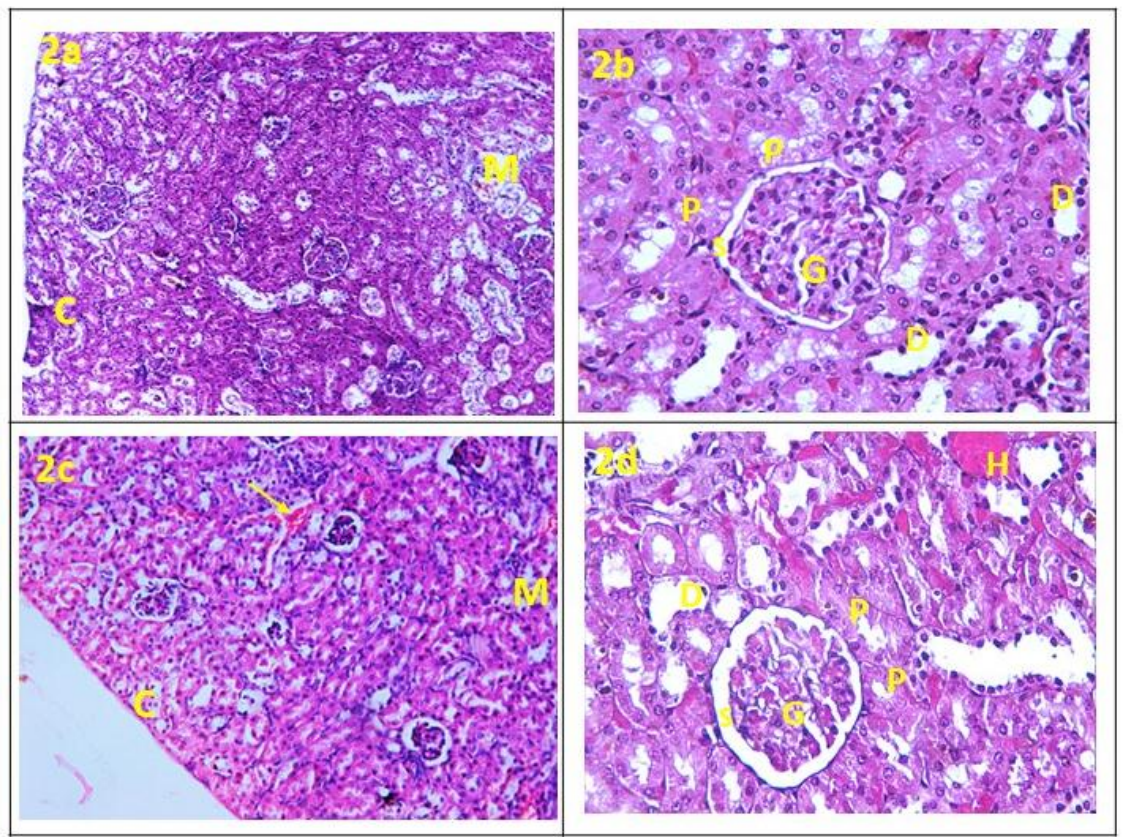

Fig. 2: Light photomicrographs of:

a: Group (3) showing outer cortex (C), inner medulla (M) (H\&E×200).

b: Group (3) showing glomeruli (G) Bowman's space (S), proximal tubules (P), distal tubules (D) $(\mathrm{H} \& \mathrm{E} \times 400)$.

c: Group (4) showing cortex (C), medulla (M), interstitial extravasation of blood (arrow) (H\&E×200).

d: Group (4) showing congested glomeruli $(\mathrm{G})$, dilated Bowman's space (S), haemorrhage $(\mathrm{H})$, dilated proximal (P), distal (D) tubules (H\&E×400).

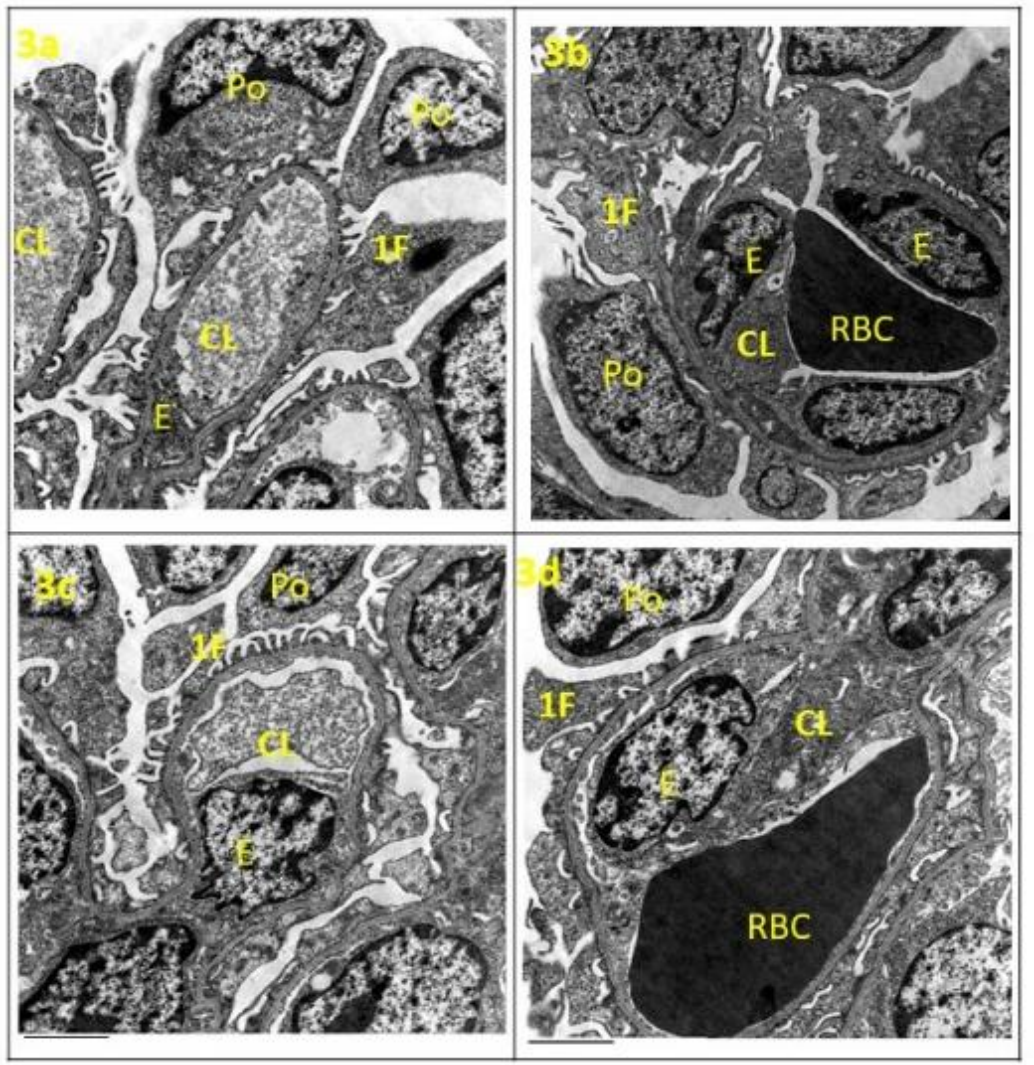

Fig. 3: Electron photomicrographs of: 
a: Group (1) showing glomerular blood capillary (CL), fenestrated cytoplasm (E), podocyte (Po) primary foot processes $(1 \mathrm{~F})$.

b: Group (2) showing glomerular blood capillary (CL) red blood cell (RBC), intended nucleus of endothelial cell (E), distorted primary foot processes $(1 \mathrm{~F})$, podocyte $(\mathrm{Po})$.

c: Group (3) showing glomerular blood capillary (CL), fenestrated cytoplasm (E), podocyte (Po), primary foot processes $(1 \mathrm{~F})$.

d: Group (4) showing glomerular blood capillary (CL), red blood cell (RBC), intended nucleus of endothelial cell (E), distorted primary foot processes $(1 \mathrm{~F})$ podocyte $(\mathrm{Po})(\mathrm{TEM} \times 12000)$

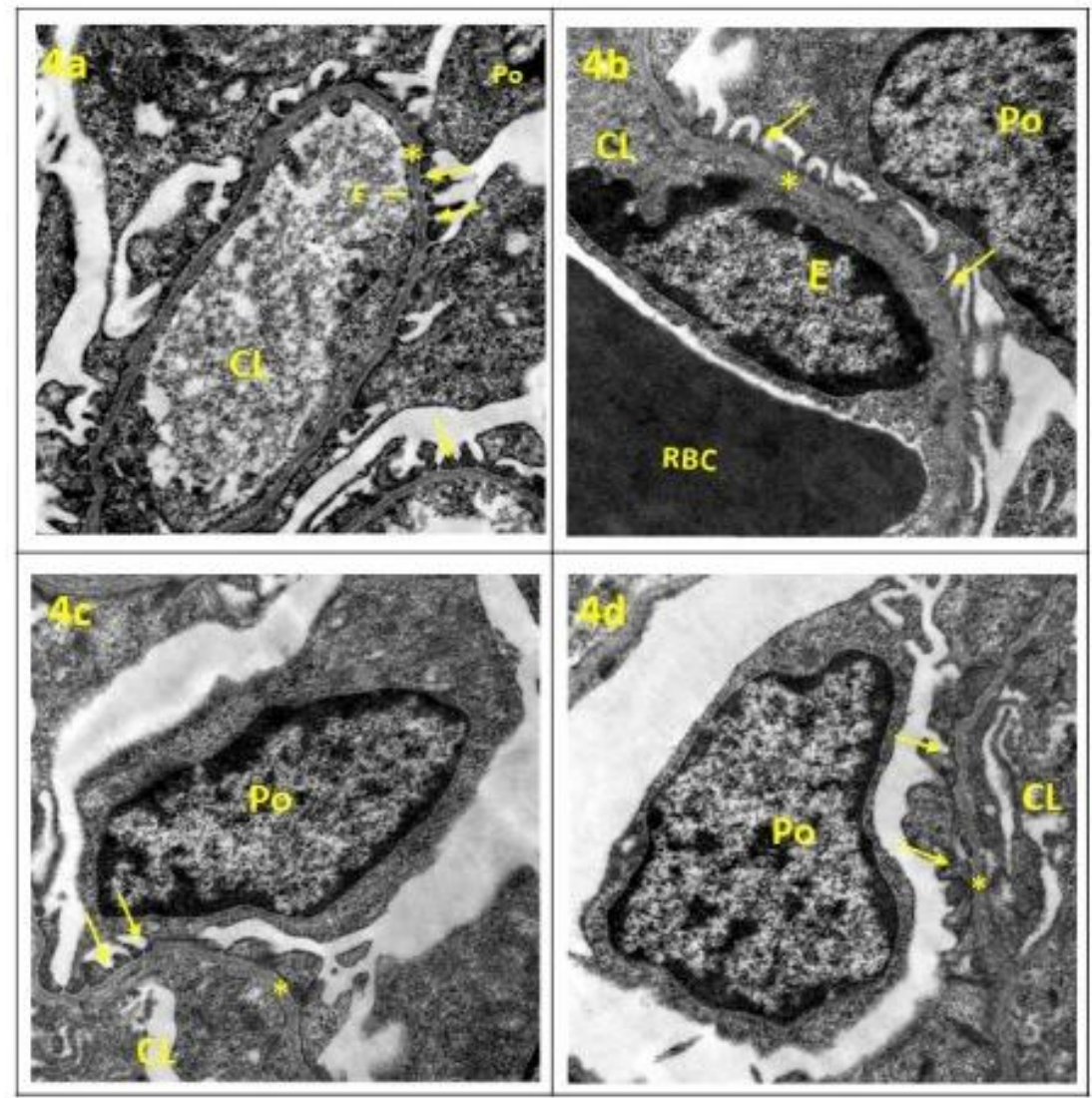

Fig. 4: Electron photomicrographs of:

a: Group (1) showing glomerular blood capillary (CL), fenestrated endothelial cytoplasm (E), glomerular basement membrane (star), filtration slits (arrows), podocyte (Po).

b: Group (2) showing glomerular blood capillary (CL), red blood cell (RBC), thickened irregular basement membrane (star), intended nucleus of endothelial cell (E), obliterated slits (arrows), podocyte (Po).

c: Group (3) showing glomerular blood capillary (CL), glomerular basement membrane (star), filtration slits (arrows) (Po).

d: Group (4) showing glomerular blood capillary (CL) thickened irregular glomerular basement membrane (star), obliterated filtration slits (arrows), podocyte (Po) (TEM x20000) 

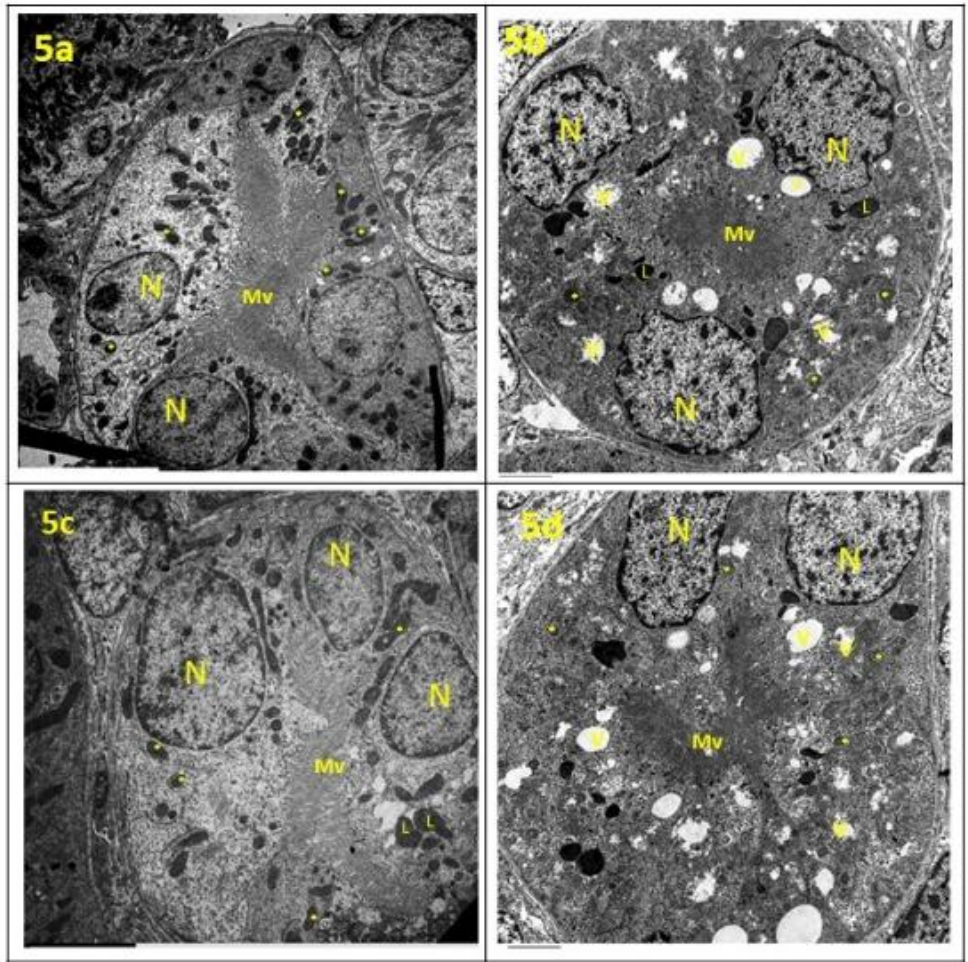

Fig. 5: Electron photomicrographs of:

a: Group (1) showing PCT cells nuclei (N), microvilli (Mv), mitochondria (stars).

b: Group (2) showing PCT, the lining cells had irregular nuclei (N), cytoplasmic vacuolations (v), microvilli (Mv), mitochondria (stars), lysosomes (L).

c: Group (3) showing PCT cells had oval nuclei(N), microvilli (Mv), mitochondria, (stars), few lysosomes (L)

d: Group (4) showing PCT cells had irregular nuclei(N), vacuolations (v), microvilli (Mv), mitochondria, (stars) lysosomes (L). (TEM x8000)
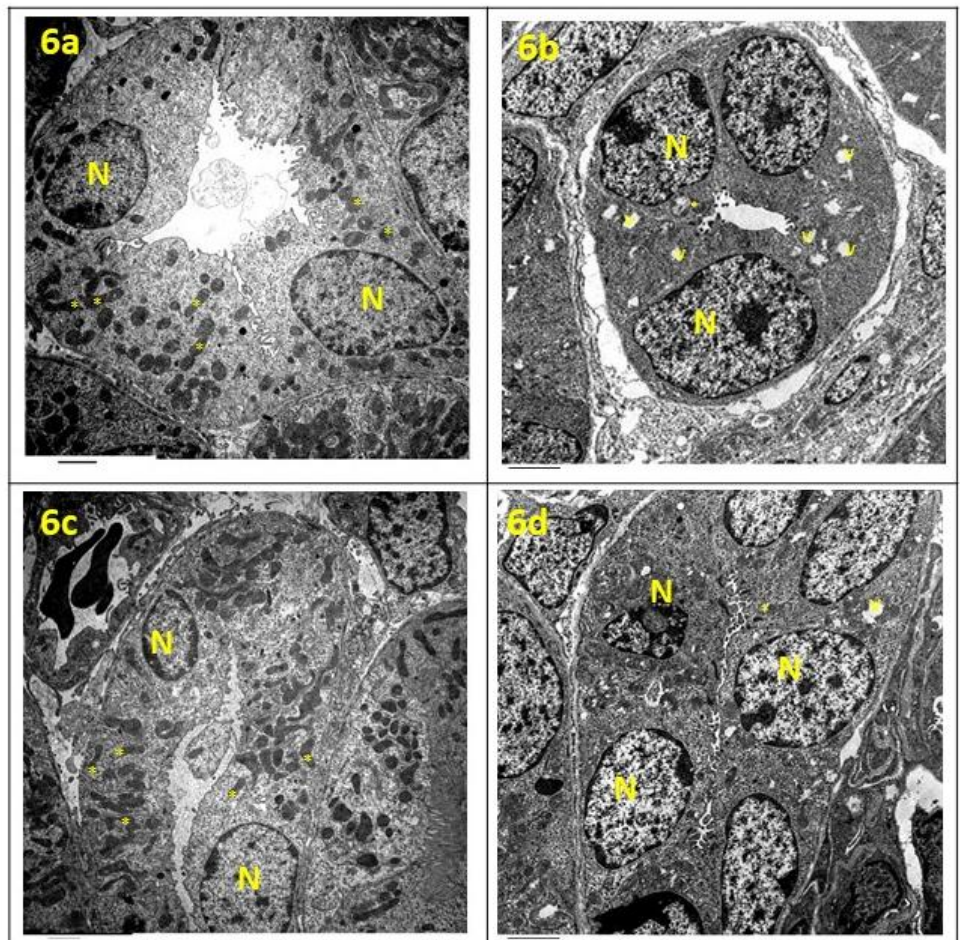

Fig. 6: Electron photomicrographs of: 
a: Group (1) showing DCT cells nuclei (N), mitochondria, (stars).

b: Group (2) showing DCT cells nuclei $(\mathrm{N})$, vacuolations (v), mitochondria (star).

c: Group (3) showing DCT cells nuclei(N), mitochondria (star).

d: Group (4) showing DCT cells nuclei $(\mathrm{N})$, vacuolations(v), mitochondria (star). (TEM x8000)

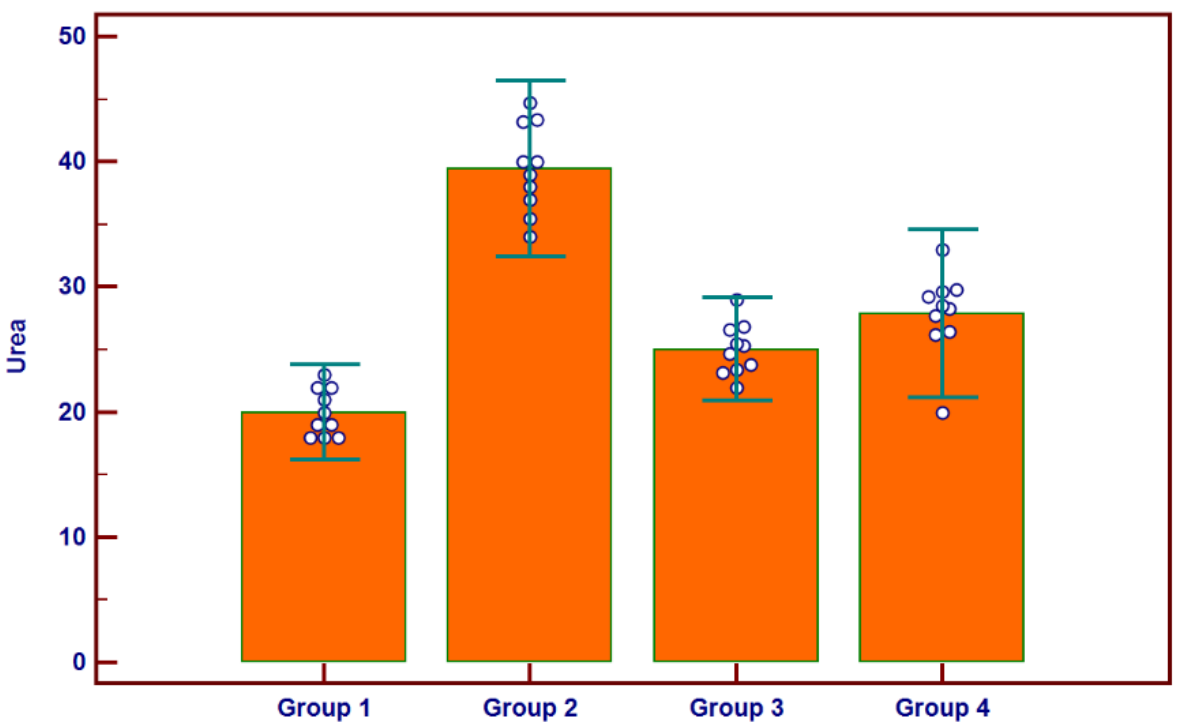

Fig. (7): Level of urea (mg/dl) in all experimental groups

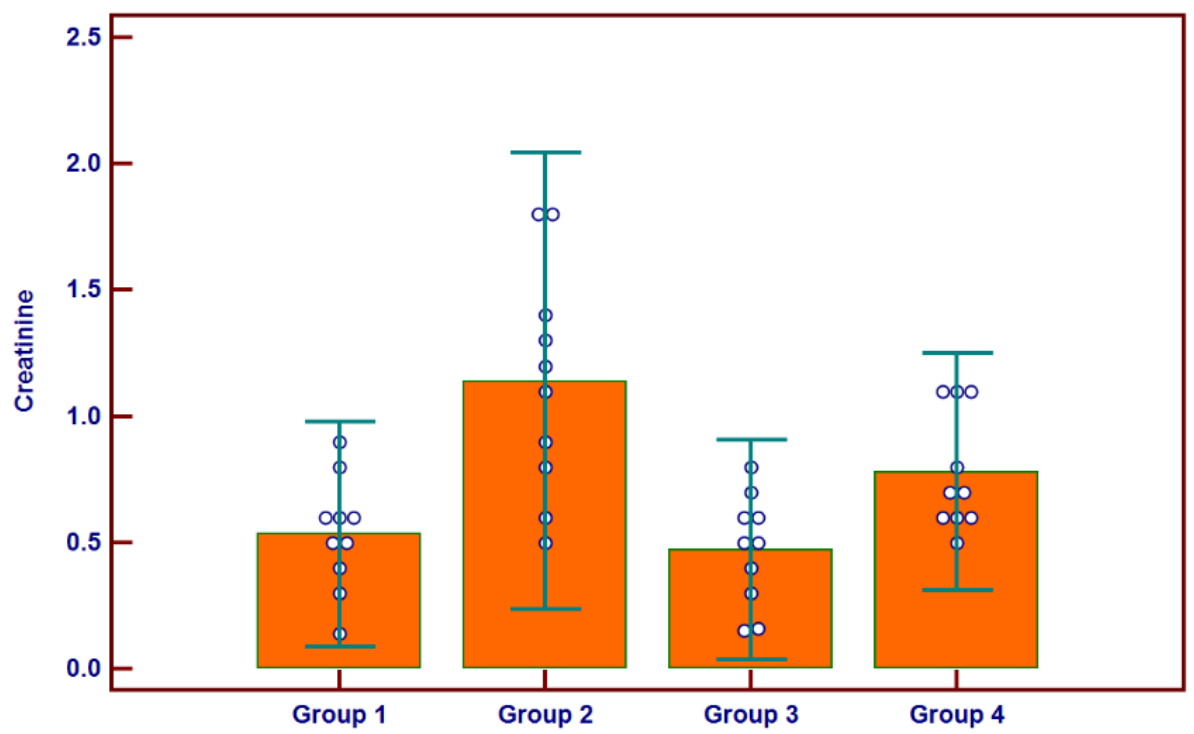

Fig. (8): Level of creatinine (mg/dl) in all experimental groups 


\begin{tabular}{|c|c|c|c|c|c|}
\hline \multirow{2}{*}{ Groups } & \multicolumn{2}{|c|}{ Urea (mg/dl) } & \multirow{2}{*}{ Test value } & \multirow{2}{*}{ P-value } & \multirow{2}{*}{ Sig. } \\
\hline & Mean \pm SD & Range & & & \\
\hline Group 1 & $20.00 \pm 1.89$ & $18-23$ & \multirow{4}{*}{86.387} & \multirow{4}{*}{0.000} & \multirow{4}{*}{ HS } \\
\hline Group 2 & $39.48 \pm 3.52$ & $34-44.7$ & & & \\
\hline Group 3 & $25.03 \pm 2.07$ & $22-29$ & & & \\
\hline Group 4 & $27.87 \pm 3.37$ & $20-33$ & & & \\
\hline \multicolumn{6}{|c|}{ Post hoc analysis using LSD test } \\
\hline $\mathrm{P} 1$ & $\mathrm{P} 2$ & P3 & $\mathrm{P} 4$ & P5 & P6 \\
\hline$<0.001$ & $<0.001$ & $<0.001$ & $<0.001$ & $<0.001$ & 0.030 \\
\hline
\end{tabular}

P1: Group 1 vs group 2

P2: Group 1 vs group 3

P3: Group 1 vs group 4

P4: Group 2 vs group 3

P5: Group 2 vs group 4

P6: Group 3 vs group 4

Table 1. Biochemical analysis of urea in all experimental groups

\begin{tabular}{|r|c|c|c|c|c|}
\hline \multirow{2}{*}{ Groups } & \multicolumn{2}{|c|}{ Creatinine (mg/dl) } & \multirow{2}{*}{ Test value } & \multirow{2}{*}{ P-value } & Sig. \\
\cline { 2 - 3 } & Mean \pm SD & Range & & & \\
\hline Group 1 & $0.53 \pm 0.22$ & $0.14-0.9$ & & \\
Group 2 & $1.14 \pm 0.45$ & $0.5-1.8$ & 10.296 & 0.000 & HS \\
Group 3 & $0.47 \pm 0.22$ & $0.15-0.8$ & & \\
Group 4 & $0.78 \pm 0.23$ & $0.5-1.1$ & & \multicolumn{2}{|c|}{ P6 } \\
\hline \multicolumn{7}{|c|}{ Post hoc analysis using LSD test } & P4 & 0.011 & 0.027 \\
\hline
\end{tabular}

P1: Group 1 vs group 2

P2: Group 1 vs group 3

P3: Group 1 vs group 4

P4: Group 2 vs group 3

P5: Group 2 vs group 4

P6: Group 3 vs group 4

Table 2. Biochemical analysis of creatinine in all experimental groups

\section{DISCUSSION}

Nephron development (nephrogenesis) continues postnatally in the rat until postnatal day 15 while the renal cortex is considered histologically mature by postnatal day $21^{14}$.

In this work, two postnatal ages were carefully chosen to detect the effect of maternal pregabalin drug during gestation (the 2nd postnatal day) and lactation (the 21st postnatal day) on the developing renal cortex.

In the present study, light microscopic examination of the renal cortex of group (1) demonstrated that the renal cortex was formed of two zones: the subcapsular nephrogenic zone and the juxtamedullary zone. The subcapsular nephrogenic zone contained immature forms of renal glomeruli while the juxtamedullary zone contained more mature renal 
corpuscles and convoluted tubules. These findings were supported by other researchers 15,16 .

The electron microscopic examination of group (1) showed podocytes with primary and secondary foot processes with filtration slits in-between. This finding was in accordance with ${ }^{17}$.

The PCT cells showed oval basal nuclei, dense apical microvilli, numerous mitochondria, and few lysosomes DCT cuboidal cells showed large oval euchromatic nuclei, numerous mitochondria and short apical microvilli and wide lumen. These findings were in agreement with ${ }^{18}$.

However, the renal cortex of group (3) revealed mature glomeruli, PCTs, and DCTs. These results in accordance with several studies ${ }^{14,18}$. These zones were present during the early neonatal period until 7 days old rats. Nephrogenesis proceeds in a centrifugal pattern and continued up to about 3 weeks of age ${ }^{19}$.

In the current work, the effect of pregabalin administration on the structure of the developing renal cortex revealed glomerular and tubulointerstitial injury at the 2nd postnatal day in group (2) and at 21 st postnatal day in group (4).

Light microscopic examination of group (2) revealed glomerular and tubulointerstitial injury. The glomeruli appeared distorted, congested. There were dilatation in the PCTs and DCTs lumina, vacuolation with exfoliation of their epithelial lining associated with haemorrhage and congestion between the renal tubules. Ultrastructurally, the podocyte showed distorted primary foot processes and effacement of its secondary foot processes. PCT cells had irregular nuclei, cytoplasmic vacuolations, and few mitochondria with many lysosomes. DCT cells showed shrunken nuclei, cytoplasmic vacuolations and few mitochondria. Light microscopic examination of group (4) revealed glomerular distortion, dilatation and vacuolar degeneration in convoluted tubules accompanied by haemorrhage and congestion. Ultrastructurally, thickening, irregularity of glomerular basement membranes, distorted primary foot processes, with broadening and fusion of secondary foot processes of podocytes. The PCTs and DCTs cells revealed nuclear degeneration with cytoplasmic vacuolation with significant elevation in serum urea and creatinine in both ages. These results were in line with the results of Badawy et al. ${ }^{20}$ who found in their study that gabapentin, an ADE similar to pregabalin, induced similar nephrotoxicity results in rat fetuses. Kriz and Lemley ${ }^{21}$ stated that the large surface area of the glomerular capillaries might render them more sensitive to damage. Macconi et al. ${ }^{22}$ suggested that foot processes effacement leads to impairment of the glomerular filtration and progressive glomerular injury. Pavenstädt et al. ${ }^{23}$ mentioned that podocytes injury could affect selective permeability of glomeruli with subsequent kidney function alteration. Moreover, a link between tubular cytoplasmic vacuolar degeneration and the increased permeability of cell membranes as primary responses to cell injury ${ }^{24}$.

In this study, significant elevation in serum urea and creatinine were observed in groups (2) and (4) if compared with the control groups, this might be explained as the elevation of serum urea and creatinine is an indicator for renal tubule injuries and impairment of kidney function ${ }^{25}$.

In the current work, the renal histopathological changes with pregabalin use during gestation (at the 2nd postnatal day) and lactation (at the 21 st postnatal day) might be due to oxidative stress and disturbance in the oxidant/antioxidant ratio. Such suggestion was supported by Kamel ${ }^{26}$ who found that pregabalin might lead to an elevation in fetal oxidative stress with high DNA damage. Although placental enzymes could play a part in fetal protection against oxidative stress ${ }^{27}$. Zaken et al ${ }^{28}$ stated that in the early stages of organogenesis the developing embryo is susceptible to high levels of oxidative stress because of its weak antioxidant defense. As regarding humans, Winterfeld et al. ${ }^{29}$ reported an association between major fetal congenital malformations and pregabalin use during pregnancy.

\section{CONCLUSION}

Pregabalin should be used with caution during pregnancy and lactation.

Declaration of conflicts of interest: No conflicts of interest.

Authorship: The author has a substantial contribution to the article.

\section{REFERENCES}

1. Perucca E. Birth defects after prenatal exposure to antiepileptic drugs. Lancet Neurolo. 2005;4: 781-86. doi: 10.1016/S1474-4422(05)70224-6.

2. Güveli BT, Rosti RÖ, Güzeltaş A, et al. Teratogenicity of antiepileptic drugs. Clinical Psychopharmacology and Neuroscience. 2017;15(1), 19-27. doi: 10.9758/cpn.2017.15.1.19

3. Tomson T, Battino D. Pharmacokinetics, and therapeutic drug monitoring of newer antiepileptic drugs during pregnancy and the puerperium. Clinical pharmacokinetics. 2007;46: 209-19. doi: 10.2165/00003088200746030-00002.

4. Blanco Tarrio E, Gálvez Mateos R, Zamorano Bayarri E, et al. Effectiveness of pregabalin as monotherapy or combination therapy for neuropathic pain in patients unresponsive to previous treatments in a Spanish primary care setting. Clinical Drug Investigation. 2013; 33:633-45. doi: 10.1007/s40261-013-0116-7

5. Tassone DM, Boyce E, Guyer J, et al. Pregabalin: a novel gamma aminobutyric acid analogue in the treatment of neuropathic pain, partial-onset seizures, and anxiety disorders. Clinical Therapeutics. 2007; 29: $26-48$ doi: 10.1016/j.clinthera.2007.01.013. 
6. Di Guilmi MN, Urbano FJ, Inchauspe CG, et al. Pregabalin modulation of neurotransmitter release is mediated by change in intrinsic activation/inactivation properties of Cav2.1 calcium channels. Journal of Pharmacology and Experimental Therapeutics. 2010; 336: 973-82. doi: 10.1124/jpet.110.172171.

7. McNamara JO. Pharmacotherapy of the Epilepsies. In: Brunton LL, Chabner BA, Knollmann BC, editors. Goodman \& Gilman's: The Pharmacological Basis of Therapeutics. 12th ed.: McGraw-Hill Medical: New York, New York. 2011.607 33.

8. Paget GC, and Barens JM. Toxicity Tests. In: Laurence, D.R., \& Bacharach, A.L., eds., Evaluation of the drug activities. 1st ed. Academic Press: London, New York 1964. pp. 1-13.

9. Bancroft JD, Gamble M. Theory, and practice of histological techniques. 6th ed. Churchill Livingstone: Philadelphia. 2008.pp.126-38.

10. Hayat MA (2000) Principles and techniques of electron microscopy: biological application. 4th ed. Cambridge University Press: Edinburgh, UK, pp 37-59.

11. Jung D, Briggs $\mathrm{N}$, Erickson $\mathrm{J}$, et al. New colorimetric reaction for end point continues flow and kinetic measurement of urea. Clinical Chemistry. 1975; 21: 11-36. PMID: 1137920.

12. Jaffe M, Bergman A, Ledyard P. Effect of detergent on kinetic Jaffe method assay for creatinine. Clinical Chemistry. 1980; 26: 1729-32.

13. Mould, R.F. Introductory Medical Statistics. Adam Hilger, Bristol, Philadelphia, USA, 1999.pp.17-22.

14. Marquez MG, Cabrera I, Serrano DJ, et al. Cell proliferation and morphometric changes in the rat kidney during postnatal development. Anatomy Embryology. 2002;205(5-6):431-40. doi: 10.1007/s00429002-0262-9.

15. Abdel-Aziz HAM, Mohamed HK. A histological study on the effect of prenatal and postnatal administration of monosodium glutamate on the developing renal cortex of male albino rats. Egypt J Histol. 2013; 36:470- 82.

16. Brown DL, Walling BE, Mattix ME. Urinary system. In: Atlas of histology of the juvenile rat. San Diego, CA: Elsevier; 2016. 395-422.

17. Neiss WF and Klehn KL. The postnatal development of the rat kidney, with special reference to the chemo differentiation of the proximal tubule. 1981; Histochemistry, 73. 251-68.

18. El- Gammal A, Ibrahim AY, Shaban SF, et al. Postnatal development of the albino rat renal cortex (Histological Study). Egyptian Journal of Histology. 2010; 33, 745- 56.

19. Friis c. Postnatal development of the pig kidney: Ultrastructure of the glomerulus and the proximal tubule. Journal of Anatomy. 1980; 130: 513-26. PMID: 7410196

20. Badawy G M, Atallah M N and Sakr S A. Ginger ameliorates the nephrotoxicity induced by gabapentin in rat fetuses. Global Scientific Journal. 2019; 7 (1) 898-916.

21. Kriz W. and Lemley K.V. The role of the podocyte in glomerulosclerosis. Current Opinion in Nephrology and Hypertension. (1999); Jul: 8(4): 489-97. doi: 10.1097/00041552-199907000-00014.

22. Macconi D, Bonomelli M, Benigni A, et al. Pathophysiologic implications of reduced podocyte number in a rat model model of progressive glomerular injury. The American Journal of Pathology. 2006;168: 42-54. doi: 10.2353/ajpath.2006.050398.

23. Pavenstädt H, Kriz W, and Kretzler M. Cell biology of the glomerular podocyte. Physiological Reviews. 2003; 83: 253-307. doi.org/10.1152/physrev.00020.2002.

24. Filiopoulos V, and Vlassopoulos D. Inflammatory syndrome in chronic kidney disease: Pathogenesis and influence on outcomes. Inflammation \& Allergy Drug Targets. 2009;8(5):369-82. doi: 10.2174/1871528110908050369.

25. Ronco C, Grammaticopoulos S, Rosner M, et al. Oliguria, creatinine, and other biomarkers of acute kidney injury. Contributions to Nephrology. (2010);164: 118-27. doi: 10.1159/000313725.

26. Kamel MA. Study on DNA damage and oxidative stress and some biochemical alterations of long-term administration of alpha-2 delta (A2- $\Delta$ ) ligand pregabalin and the possibility of Zingiber officinale in ameliorating these effects in rats. World Journal of Pharmaceutical Research. 2016; 5:1528- 45.

27. Foster W, Myllynen P, Winn LM, et al. Reactive oxygen species, diabetes and toxicity in the placenta: a workshop report. Placenta. 2008; 29: S105 -S7. doi: 10.1016/j.placenta.2007.10.014.

28. Zaken V, Kohen R, and Ornoy A. The development of antioxidant defence mechanism in young rat embryos in vivo and in vitro. Early Pregnancy. 2000; 4:110 - 23. PMID: 11723541.

29. Winterfeld U, Melob P, Baud D, et al. Pregnancy outcome following maternal exposure to pregabalin may call for concern. Neurology. 2016; 86:2251-7. doi: 10.1212/WNL.0000000000002767. 